Reprinted with permission from: Leafy Spurge Symposium, Brandon, Manitoba, Canada, Aug. 13-15, 1996, p. 21.

Sponsored by: Manitoba Weed Supervisors Association and Manitoba Agriculture.

\title{
2,4-D reversal of DFMO-induced inhibition of adventitious root formation in leafy spurge (Euphorbia esula L.) in vitro
}

\author{
DAVID G. DAVIS
}

U. S. Department of Agriculture, Agricultural Research Service, Biosciences Research Laboratory, State University Station, Fargo, North Dakota 58105-5674 USA.

Indoleacetic acid (IAA) stimulates adventitious root formation and partially reverses the effects of difluoromethylornithine (DFMO), a specific suicide inhibitor of putrescine biosynthesis, in etiolated aseptically grown hypocotyl segments of the perennial weed, leafy spurge. DFMO also is a strong inhibitor of adventitious root formation in the hypocotyl segments. Dichlorophenoxyacetic acid (2,4-D) has auxin-like activity and was also tested to determine if it could counteract DFMO-induced inhibition of root formation. When 2,4-D was applied at 45 to $450 \mathrm{nM}$ in the nutrient medium, root formation was inhibited. Unexpectedly, $450 \mathrm{nM}$ 2,4-D applied simultaneously with $500 \mathrm{pM}$ DFMO reversed the DFMO-induced inhibition of adventitious root formation, forming root numbers similar to controls. This reversal occurred under growth conditions with complete B5 nutrient medium or with B5 medium containing salts and vitamins reduced to $10 \%$ of their normal concentrations. This action of 2,4-D resembles that of indoleacetic acid (IAA), although 2,4-D did not stimulate root formation in the absence of DFMO, as did IAA (at 230 to $1100 \mathrm{nM}$ ). 